\title{
MODELADO PARA OPERACIÓN ÓPTIMA DE UN SISTEMA DE REFRIGERACIÓN INDUSTRIAL
}

\author{
María P. Marcos, José Luis Pitarch, César de Prada \\ Instituto de Procesos Sostenibles, Dpto. Ingeniería de Sistemas y Automática, Universidad de Valladolid. \\ C/Real de Burgos s/n, 47011, Valladolid, \{maria.marcos | jose.pitarch | prada\}@autom.uva.es \\ Christian Jasch
}

LENZING AG. Werkstraße 2, 4860, Lenzing, Austria, c.jasch@lenzing.com

\section{Resumen}

Este trabajo trata el problema de modelado y distribución óptima de agua en una red de refrigeración asociada a plantas de evaporación. El fin último es desarrollar una herramienta de optimización en tiempo real para balancear el uso de agua con el consumo energético de las plantas (vapor vivo). El problema de optimización incluye modelos experimentales del impacto que tiene la operación del equipo de refrigeración en la eficiencia global de las plantas. Se ha propuesto una metodología eficaz de modelado para optimización, de forma que se asegure la coherencia física de los modelos con el proceso. Además, la herramienta proporciona una estimación periódica de la pérdida de eficiencia en los equipos debido a una operación continuada (ensuciamiento en intercambiadores de calor).

Palabras clave: modelado robusto, red de evaporación, distribución de agua, modelos empíricos, RTO.

\section{INTRODUCCIÓN}

En la industria de procesos existe una cada vez mayor conciencia sobre la necesidad de producir de la forma más eficiente posible. Para ello, en las plantas se ha de tener en cuenta tanto la situación de la producción en tiempo real, como la eficiencia energética y de recursos global. A todo esto hay que añadir la regulación cada vez más restrictiva en materia medioambiental. En consecuencia, para que una empresa se mantenga competitiva en un mercado global, se han de tomar múltiples acciones que conduzcan a un ahorro en el consumo de energía y recursos, entre las cuales se incluye la toma de decisiones de operación en tiempo real [1].

Con el objetivo de facilitar la toma de decisiones a los operarios y a los jefes de planta, se les ha de proveer de una herramienta automática que les proporcione dicho conjunto de decisiones óptimas en un breve periodo de tiempo [2, 3]. Generalmente, para desarrollar estas herramientas se han de tener en cuenta las características de la planta (desarrollando modelos), la comunicación con los sistemas de control e información de la factoría, y los algoritmos de optimización disponibles.

Así pues, en este trabajo se ha desarrollado una herramienta que realiza una optimización en tiempo real (RTO, por sus siglas en inglés) del sistema de refrigeración en una red de evaporadores industriales perteneciente a la empresa Lenzing AG (Austria), referencia mundial en la producción de fibra viscosa textil. El objetivo de esta optimización es distribuir el agua de refrigeración entre los condensadores de las diferentes plantas, de forma que se minimice el coste del consumo de vapor vivo y de la propia agua utilizada (recursos). Dicha optimización se ha programado usando CasADi [4] en MATLAB, y posteriormente se ha vinculado con el sistema de información de la planta [5].

La optimización está basada en modelos experimentales obtenidos a partir de diferentes test que se han realizado con el sistema de refrigeración. En concreto se han modelado dos de los aspectos clave que se han de tener en cuenta en el proceso: la temperatura de salida del agua del sistema de refrigeración, puesto que va a parar al río y debido a las restricciones medioambientales no ha de superar un cierto valor; y la variación del consumo específico de vapor que tiene la planta de evaporación debida a la operación del sistema de refrigeración. Sin embargo, el ajuste de modelos tipo caja negra a datos debe realizarse con cautela, ya que asegurar la robustez frente a medidas corruptas y la coherencia con la física del proceso son aspectos fundamentales [6], habitualmente olvidados y anegados por la vorágine de aplicaciones Industria 4.0 y "big data".

El resto del trabajo se organiza como sigue. La siguiente sección describe brevemente el caso de estudio de forma general y la red de refrigeración en particular, cuya operación se quiere optimizar. En la Sección 3 se plantean los modelos experimentales que se van a utilizar. La Sección 4 presenta la formulación del problema de optimización (distribución óptima de agua). En la Sección 5 se analiza el comportamiento de los modelos planteados y se propone una metodología eficaz de modelado para optimización. La Sección 6 presenta resultados y 
recomendaciones arrojadas para una situación real de la red en un instante determinado. Por último, la Sección 7 expone las conclusiones generales junto con las líneas de trabajo futuras.

\section{DESCRIPCIÓN DEL SISTEMA}

La planta con la que se trabaja es una fábrica de producción de fibra viscosa textil obtenida a partir de madera. Una vez triturada, la pulpa de celulosa que contiene la madera se trata químicamente y se convierte en una solución viscosa. La etapa clave de la producción es el hilado, donde se produce la reconversión de dicha solución en nuevas fibras haciéndola pasar a presión por una especie de tamices de fino diámetro e introduciéndola en un baño ácido. Aparte de las nuevas fibras sólidas, como parte del mencionado tratamiento se produce sulfato de sodio $\left(\mathrm{Na}_{2} \mathrm{SO}_{4}\right)$ y agua como subproductos, degradando así la acidez del baño y, en consecuencia, la calidad del producto obtenido. Por lo tanto, se hace necesario regenerar dicho baño mediante la extracción continua de agua y $\mathrm{Na}_{2} \mathrm{SO}_{4}$. A tal efecto se utiliza una red de plantas de evaporación y una sección de cristalización, anexas al proceso de producción principal.

La red de evaporación está formada por 20 plantas de evaporación con capacidades nominales dispares. Esta red debe ser capaz de regenerar los baños ácidos provenientes del hilado, existiendo varios tipos de fibras a fabricar. La eficiencia de las plantas de evaporación depende de diferentes factores: la carga de evaporación (EC), las condiciones de operación (temperatura y caudal del producto), la eficacia del sistema de refrigeración y el grado de ensuciamiento en los diferentes equipos. Para una explicación más detallada de las plantas de evaporación así como de su sistema de control, se remite al lector consultar $[7,8]$.

Las plantas de evaporación pueden tener dos sistemas de refrigeración: por condensadores de superficie (SC) (véase Figura 1) o mediante torres de refrigeración. De los 20 totales, existen $\mathcal{E}=15$ evaporadores equipados con $\mathrm{SC}$ como sistemas de refrigeración, que, a su vez, se pueden agrupar en dos subredes según su disposición física en la planta y la fuente de la que se obtiene el agua de refrigeración (véase Figura 2). Estos condensadores son los encargados de succionar y condensar el vapor procedente de las plantas.

Puesto que el agua total disponible de cada fuente de alimentación para usar como refrigerante es limitada, su uso implica un coste, el cual viene dado por la negociación con otros departamentos de la factoría. Sin embargo, cuanta más agua de

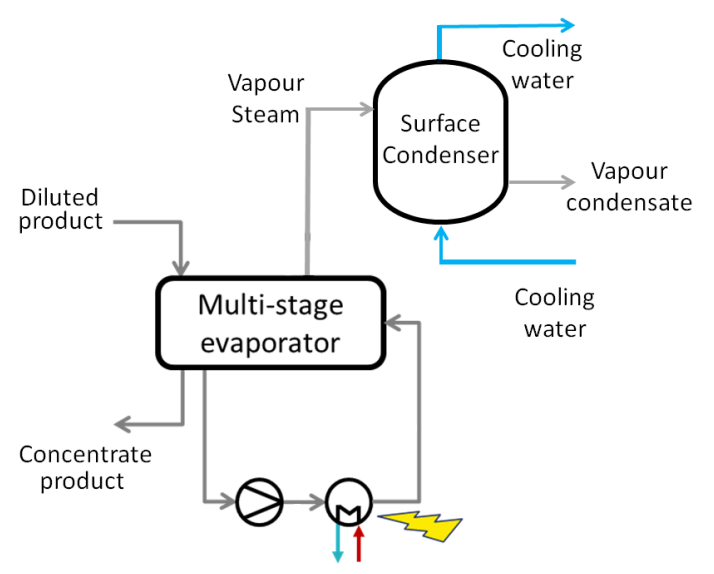

Figura 1: Esquema simplificado de una planta de evaporación.

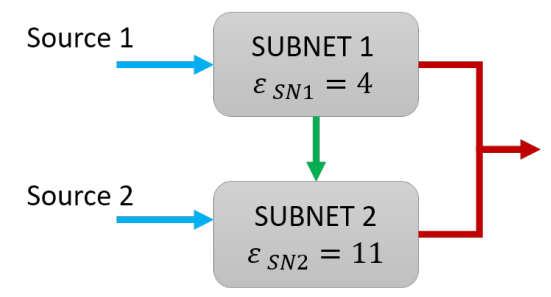

Figura 2: Red de condensadores de superficie

refrigeración alimente a los condensadores de superficie, más eficientes son las plantas de evaporación, puesto que se reduce el consumo específico de vapor vivo. Por tanto, el problema de la distribución de agua de refrigeración viene dado por el compromiso entre el coste del consumo energético (consumo específico de vapor) y el coste del consumo de recursos (agua de refrigeración). Así pues, el objetivo es determinar la distribución óptima de agua para cada subred, teniendo en cuenta ciertas limitaciones.

\section{MODELADO DEL SISTEMA}

Con el objetivo de estudiar el efecto de la operación de los SCs en el comportamiento de la planta, se han hecho diferentes experimentos y se han recolectado los datos obtenidos. A partir de dichos datos se han elaborado modelos experimentales de tipo caja negra con los que predecir el efecto del sistema de refrigeración en el consumo de vapor vivo de las plantas.

\subsection{DISEÑO DE EXPERIMENTOS}

En los test experimentales realizados, se han alimentado los SCs con diferentes caudales cubriendo su rango de operación habitual y se han recogido los datos de temperatura de salida del agua y del consumo de vapor específico que tiene la planta 
de evaporación asociada al SC. Sin embargo, para la correcta identificación de modelos según la propuesta presentada en las siguientes secciones, se han de tener en cuenta las siguientes consideraciones a la hora de diseñar los tests:

- La carga de trabajo de la planta, i.e. capacidad evaporación (EC), debe ser fija durante los tests.

- La ventana temporal en la que se realizan los test debería ser de unas pocas horas. De esta forma se asegura que la temperatura de entrada de agua al sistema de refrigeración sea aproximadamente constante.

- Los test deben planificarse a continuación de realizar una tarea de limpieza en los SCs. Esto es crucial para que el estado de ensuciamiento de los SC no afecte a las medidas de consumo especifico de vapor.

Estas consideraciones permitien que los datos obtenidos puedan combinarse y/o completarse con los de experimentos posteriores (necesaria actualización de modelos con el tiempo). Además, se asegura que el mínimo consumo específico de vapor que pueden tener los evaporadores sea el mínimo recogido por los experimentos (dato de utilidad para la obtención del modelo que predice el consumo específico de vapor la Sección, véase la Sección 3.3).

\subsection{MODELO DE TEMPERATURA DEL AGUA}

En primer lugar se han recogido datos de la temperatura de agua de refrigeración a la entrada $T_{\text {in }}^{\mathrm{e}}$ y a la salida $\mathrm{T}_{\text {out }}^{\mathrm{e}}$, en función del caudal de agua $F$ enviado a cada SC. Como resultado se puede ajustar una curva $\Delta \mathrm{T}=f(F)$ que relacione la diferencia de temperatura con el caudal de agua (véase Figura 3). Se ha visto que polinomios de grado no superior a 3 son suficientes para conseguir un buen ajuste ${ }^{1}$ :

$$
\Delta \mathrm{T}=\alpha_{0}+\alpha_{1} F+\alpha_{2} F^{2}+\alpha_{3} F^{3}, \quad \alpha_{i} \in \mathbb{R}
$$

De esta forma, la temperatura de salida del agua de refrigeración se podría calcular en cualquier momento, proporcionando la medida de la temperatura de entrada en tiempo real $\hat{\mathrm{T}}_{\mathrm{in}}$ :

$$
\overline{\mathrm{T}}_{\text {out }}=\Delta \mathrm{T}+\hat{\mathrm{T}}_{\text {in }}
$$

Además, asumiendo que los experimentos se llevaron a cabo con los SCs totalmente limpios, cuando

\footnotetext{
${ }^{1}$ Detalles sobre la metodología en la Sección 5.
}

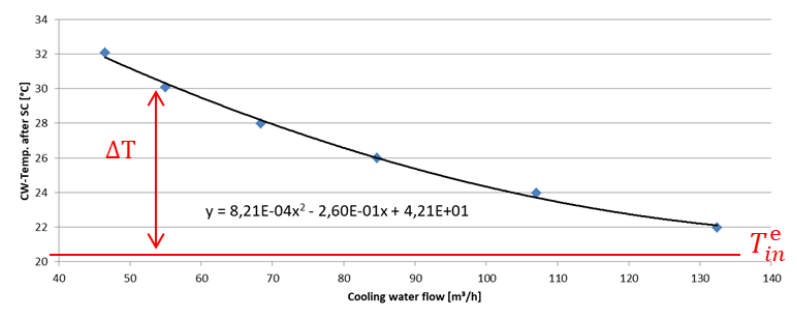

Figura 3: Modelo experimental $\Delta \mathrm{T}=f(F)$

estos se ensucien y parte de las partículas se adhieran a las paredes de los condensadores, disminuirá la transmisión de calor y por tanto la temperatura de salida del agua de refrigeración será menor. Es decir, la temperatura medida a la salida será menor a la predicha por el modelo, tal y como muestra la Figura 4 (marcador rojo).

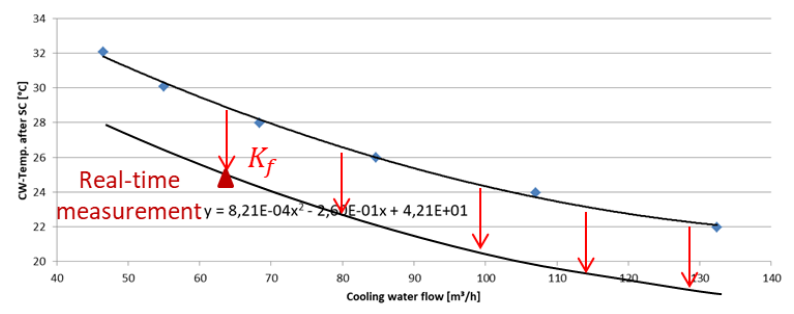

Figura 4: Adaptación del modelo al estado de ensuciamiento

Por consiguiente, se ha propuesto añadir un parámetro $\mathrm{K}_{\mathrm{f}}$ al modelo, que actúa como un "bias" para desplazar la curva base obtenida de los experimentos a la medida en tiempo real.

$$
\mathrm{T}_{\text {out }}=\Delta \mathrm{T}+\hat{\mathrm{T}}_{\mathrm{in}}-\mathrm{K}_{\mathrm{f}}
$$

De esta forma se tiene en cuenta el estado de ensuciamiento en cada $\mathrm{SC}$ a la hora de realizar la optimización, y el parámetro $K_{\mathrm{f}}$ puede ser actualizado fácilmente a partir de la temperatura de salida medida en tiempo real ( $\left.\hat{\mathrm{T}}_{\text {out }}\right)$ según:

$$
K_{f}=\left(\Delta T+\hat{T}_{\text {in }}\right)-\hat{T}_{\text {out }}
$$

\subsection{MODELO DE CONSUMO ESPECÍFICO DE VAPOR}

Por otra parte, a partir de los datos recolectados de temperatura entrada-salida y caudal volumétrico de agua, se puede calcular la potencia de enfriamiento $\left(\mathrm{C}_{\text {pow }}\right)$ del $\mathrm{SC}$ según:

$$
\mathrm{C}_{\text {pow }}=\frac{4,18}{3600} F \cdot\left(\hat{\mathrm{T}}_{\text {out }}-\hat{\mathrm{T}}_{\text {in }}\right)
$$

Además, se recogieron medidas del consumo específico de vapor (SSC) en la planta, por lo que se puede obtener una relación $\mathrm{SSC}=g\left(\mathrm{C}_{\text {pow }}\right)$ entre dicho consumo y la potencia de enfriamiento 
alcanzada en el SC. Por inspección visual también se ha determinado que polinomios de grado bajo consiguen buenos ajustes (véase Figura 5):

$$
\mathrm{SSC}=\beta_{0}+\beta_{1} \mathrm{C}_{\text {pow }}+\beta_{2} \mathrm{C}_{\text {pow }}^{2}+\beta_{3} \mathrm{C}_{\text {pow }}^{3}, \beta_{i} \in \mathbb{R}
$$

Sin embargo, se tiene que eliminar la dependencia del modelo base con el punto de operación de la planta en los experimentos (asumida una carga fija durante los mismos). Para ello, la idea es determinar el mejor consumo de vapor específico (BSSC), es decir, el mínimo dentro del rango de operación posible y determinar, de nuevo, un modelo incremental:

$$
\Delta \mathrm{SSC}=g\left(\mathrm{C}_{\text {pow }}\right)-\mathrm{BSSC}
$$

Para que esto sea cierto, se hacen dos suposiciones:

- Las pruebas se realizaron con los SC limpios.

- El modelo para $\Delta$ SSC (por ejemplo, la forma de la curva de la Figura 5), no varía significativamente de un punto de operación a otro.

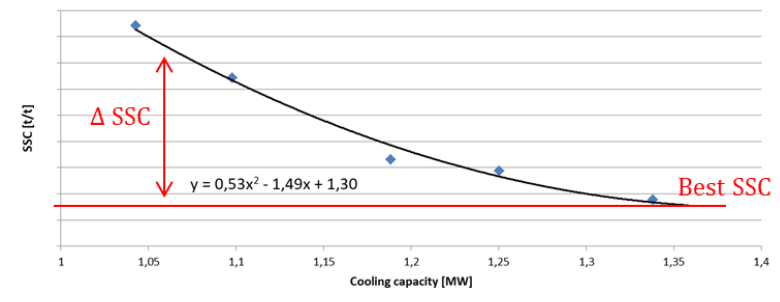

Figura 5: Modelo experimental $\mathrm{SSC}=g\left(\mathrm{C}_{\text {pow }}\right)$

\section{FORMULACIÓN DEL PROBLEMA RTO}

Tal y como se ha comentado anteriormente, la red de evaporadores con SC se puede dividir en dos subredes según la fuente de agua de refrigeración: la primera (SN1) compuesta por $\mathcal{E}_{S N 1}=4$ evaporadores y la segunda (SN2) incluye los otros $\mathcal{E}_{S N 1}=11$. Se ha de tener en cuenta que los SC de cada subred están conectados en paralelo, así como que la SN2 puede recibir agua de la SN1 pero no a la inversa. La distribución optima depende de la eficiencia de cada planta, es decir, del incremento de consumo de vapor específico ( $\Delta \mathrm{SSC})$, determinado por la carga del evaporador EC y el estado de ensuciamiento del condensador $\mathrm{K}_{\mathrm{f}}$.

Teniendo en cuenta la distribución de la red y los aspectos clave para obtener la máxima eficiencia del proceso, se ha formulado el problema de optimización que da como resultado el caudal de agua de refrigeración óptimo que debe tener cada SCs. El objetivo es encontrar la distribución de agua que minimice el compromiso entre el incremento en el consumo de vapor vivo (ASC) ponderado por su coste económico $\left(\mathrm{P}_{\text {steam }}\right)$, y de agua de refrigeración utilizada $(F)$ por su coste estimado $\left(\mathrm{P}_{\text {water }}\right)$.

Las restricciones del problema son:

- El total de agua demandada debe ser menor que el máximo disponible en las fuentes.

- La subred SN1 puede trasvasar agua a la SN2 pero no a la inversa.

- Existen límites en los caudales de agua de refrigeración para cada $\mathrm{SC}$, denotado por el subíndice $e,\left(\underline{\mathrm{F}_{e}}, \overline{\mathrm{F}_{e}}\right)$.

- La temperatura de agua de refrigeración a la salida de los SCs debe ser menor que la máxima permitida $\left(\mathrm{T}_{\max }\right) .^{2}$

Así pues, el problema se formula como sigue:

$$
\begin{aligned}
\min _{F_{e}, F_{N 12} \in \mathbb{R}^{\mathcal{E}+1}} & \sum_{e=1}^{\mathcal{E}}\left(\mathrm{ASC}_{e} \cdot \mathrm{P}_{\text {steam }}+F_{e} \cdot \mathrm{P}_{\text {water }}\right) \\
\text { s. a.: } & \sum_{e=1}^{\mathcal{E}_{S N 1}} F_{e}+F_{N 12} \leq \mathrm{F}_{\mathrm{S} 1} \\
& \sum_{e=1}^{\mathcal{E}_{S N 2}} F_{e}-F_{N 12} \leq \mathrm{F}_{\mathrm{S} 2} \\
& F_{N 12} \geq 0 \\
& \frac{\mathrm{F}_{e} \leq F_{e} \leq \overline{\mathrm{F}_{e}} \quad \forall e \in \mathcal{E}}{\mathrm{T}_{\text {out }_{e}} \leq \mathrm{T}_{\max } \quad \forall e \in \mathcal{E}}
\end{aligned}
$$

Donde $F_{N 12}$ es el caudal de agua que se trasvasa de SN1 a SN2, y el ASC se obtiene a partir del consumo específico por la carga de cada planta:

$$
\mathrm{ASC}_{e}=\Delta \mathrm{SSC}_{e} \cdot \mathrm{EC}_{e}
$$

Aquí $\mathrm{T}_{\text {out }} \mathrm{y} \Delta \mathrm{SSC}$ se obtienen mediante los modelos experimentales (3) y (7) obtenidos para cada planta, respectivamente.

Teniendo en cuenta que los modelos experimentales son polinomios en función de las variables de decisión $F_{e}$, este problema de optimización se puede resolver en tiempo real mediante algoritmos eficientes de programación no lineal (NLP).

\section{METODOLOGÍA DE MODELADO}

Para obtener los modelos (1) y (7) a partir de los datos recogidos en planta, uno podría estar tenta-

\footnotetext{
${ }^{2}$ Esta última restricción se debe a que el agua a la salida va a parar al río, debiendo cumplir la legislación medioambiental en vigor.
} 
do a seguir el enfoque clásico de ajustar una curva polinomial a los datos (utilizando algún criterio de ajuste como mínimos cuadrados) de manera independiente para $f(F)$ y $g\left(\mathrm{C}_{\text {pow }}\right)$.

A la vista de los datos, polinomios de grado no superior a 3 son suficientes para conseguir "buenos" ajustes, como se observa en las figuras 3 y 5 . A priori, podría parecer que estos modelos representan bien al sistema en el rango de operación y son adecuados para la optimización. Sin embargo, como se ilustra a continuación, los resultados distan mucho de la realidad.

Las variables de decisión para la optimización de la distribución de agua son precisamente los caudales $F$ a enviados a cada SC. Así, el problema de optimización formulado en la Sección 4 utiliza la combinación de los modelos para predecir un valor de consumo de vapor $\Delta \mathrm{SSC}$ a partir del caudal propuesto $F$, en la forma:

$$
\Delta \mathrm{SSC}=g\left(\frac{4,18}{3600} F \cdot\left(f(F)-\mathrm{T}_{\mathrm{in}}^{\mathrm{e}}-\mathrm{K}_{\mathrm{f}}\right)\right)
$$

Nótese que si $\operatorname{gr}(f) \leq \phi$ y $\operatorname{gr}(g) \leq \gamma$, el polinomio (10) es de grado menor o igual a $\gamma \cdot(\phi+1)$ en $F$. En consecuencia, ligeras desviaciones en los datos experimentales debidas a perturbaciones o ruido de medida (inapreciables en primera instancia) pueden derivar en modelos con comportamientos inesperados e incoherentes con la física del proceso. Por ejemplo, las figuras 6 y 7 muestran un caso donde un mejor ajuste clásico de $f$ y $g$, polinomios de grado 3 (en azul) frente a grado 2 (en rojo), resulta en un comportamiento inaceptable en (10): en concreto en la región resaltada se observa una disminución de la potencia de refrigeración a caudales de agua elevados, con el correspondiente incremento de consumo específico de vapor, lo cual es incoherente por las leyes físicas. Además, esto se transmite directamente a la optimización, provocando la aparición de un mínimo local inexistente en la realidad.

Observación. Se ha de destacar que dicho comportamiento incoherente no sería detectado siguiendo la metodología clásica de modelado, ya que los datos experimentales tanto de temperatura respecto al caudal (gráfica superior de la Figura 6) como de consumo específico de vapor (Figura 7) respecto a la potencia de refrigeración no muestran puntos fuera de tendencia. Sin embargo, la medida resaltada en la Figura 6 si muestra una desviación incoherente al computar el SSC respecto a $F$.

Por otro lado, utilizando polinomios de grado 2 para ajustar los modelos base se obtiene un comportamiento coherente, aunque las capacidades de extrapolación del modelo puedan seguir estando comprometidas debido a la ausencia de datos ex-
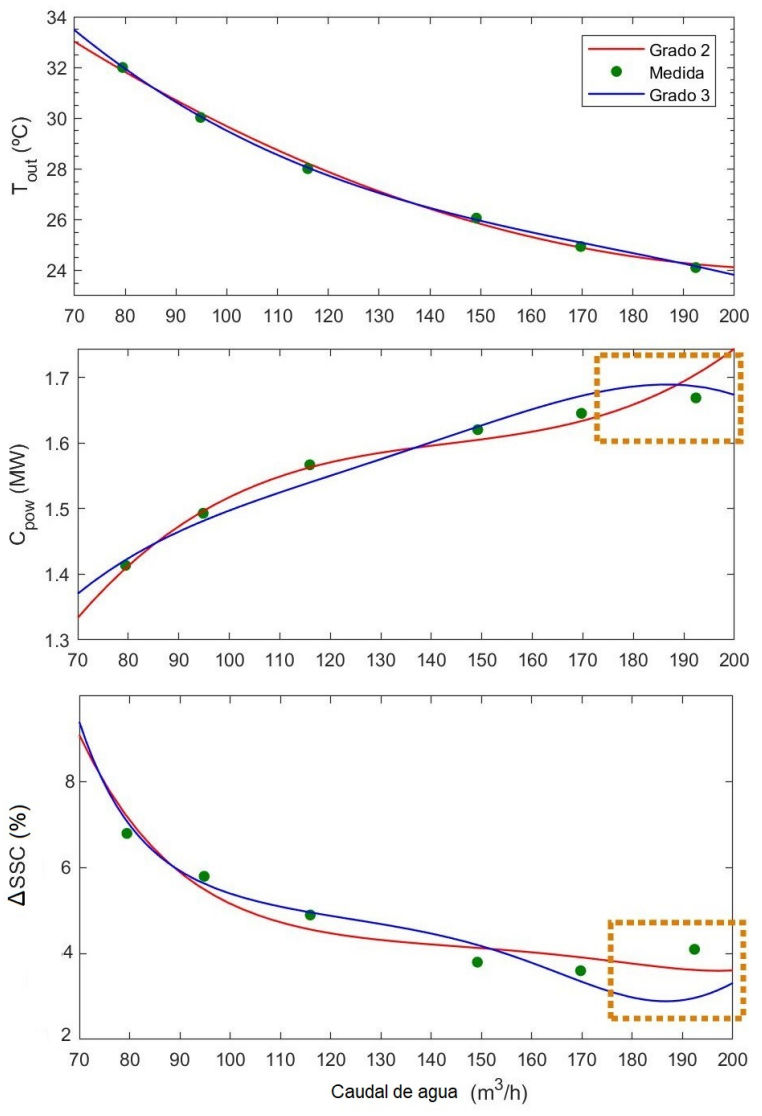

Figura 6: Comportamiento respecto al caudal $F$.

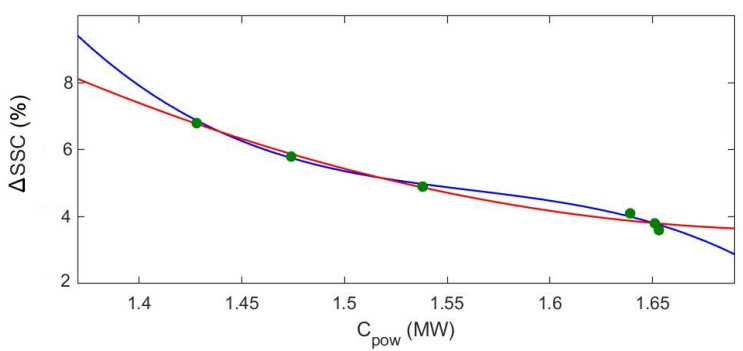

Figura 7: Modelo SSC vs $\mathrm{C}_{\text {pow }}$.

perimentales fuera del rango donde los experimentos fueron realizados.

El análisis anterior resalta la necesidad de proponer una metodología de modelado para optimización, distinta de la clásica. La propuesta se basa en las ideas recogidas en [9] y consiste de dos pasos:

1) Ajuste. Dada una serie de $N$ datos $(\Delta \mathrm{T}$, $\left.\Delta \mathrm{SSC}, \mathrm{C}_{\text {pow }}, F\right)$ ordenada de menor a mayor caudal $F$, encontrar el mejor ajuste polinomial para $f$ y $g$ en (10) resolviendo el siguiente problema de optimización mixta-entera no lineal, donde la función objetivo sigue el criterio de corregido de Akaike [10] por el cual se busca un compromiso entre complejidad de modelos (determinado por 
la variable $D$ ) y el error de ajuste.

$$
\begin{aligned}
& \text { mín } \\
& \alpha, \beta, D \in \mathbb{R}^{9} \\
& y \in\{0,1\}^{4} \\
& N \log \left(N ^ { - 1 } \left(J_{\Delta \mathrm{T}}+J_{\mathrm{C}_{\text {pow }}}+\right.\right. \\
& \left.\left.J_{\Delta \mathrm{SSC}}\right)\right)+2 D+\frac{2 D(D+1)}{N-D-1} \\
& \text { s.t. } y_{1}+y_{2}+y_{3}+y_{4} \leq D \\
& \alpha^{\mathrm{l}} y_{1} \leq \alpha_{2} \leq \alpha^{\mathrm{u}} y_{1}, \alpha^{\mathrm{l}} y_{2} \leq \alpha_{3} \leq \alpha^{\mathrm{u}} y_{2} \\
& \beta^{\mathrm{l}} y_{3} \leq \beta_{2} \leq \beta^{\mathrm{u}} y_{3}, \beta^{\mathrm{l}} y_{4} \leq \beta_{3} \leq \beta^{\mathrm{u}} y_{4} \\
& g\left(\mathrm{C}_{\text {pow }_{i+1}}\right)-g\left(\mathrm{C}_{\text {pow }_{i}}\right)<0 \quad i \in \mathcal{N} \\
& f\left(F_{i+1}\right)-f\left(F_{i}\right)<0 \quad i \in \mathcal{N} \\
& g \circ f\left(F_{i+1}\right)-g \circ f\left(F_{i}\right)<0 \quad i \in \mathcal{N}
\end{aligned}
$$

Donde $\mathcal{N}:=\{1, \ldots, N-1\}, g \circ f(F)$ se calcula con (10) y $J$ computa las diferencias entre modelo y medidas utilizando la función "fair":

$$
J:=C^{2} \sum_{i=1}^{N}\left(\frac{\left|\epsilon_{i}\right|}{C}-\log \left(1+\frac{\left|\epsilon_{i}\right|}{C}\right)\right)
$$

Siendo $C \in \mathbb{R}^{+}$el parámetro que define la sensibilidad de la función fair ante medidas incoherentes, y $\epsilon_{i}$ se obtiene en cada caso según:

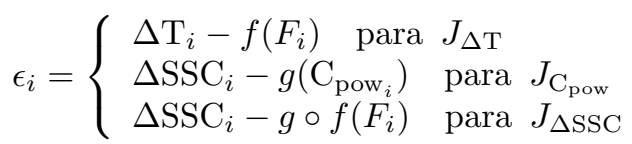

Con respecto a las restricciones del problema, nótese que la variable de decisión $D$ en (11a) limita la complejidad del modelo, i.e., la aparición de términos cuadráticos y/o cúbicos en $f$ y $g$, mediante las variables binarias $y$ las cuales, mediante (11b) y (11c), permiten anular adecuadamente ciertos parámetros $\alpha$ y $\beta$ de los modelos $f$ y $g$. No obstante, la aportación fundamental se encuentra en la imposición de restricciones adicionales sobre el modelo en el problema de ajuste (11d)-(11f) donde se indica que la derivada numérica de las tres funciones ha de ser negativa puesto que físicamente a mayor caudal, menor temperatura de salida y menor consumo de vapor.

2) Validación. Obtenidos los parámetros $\alpha$ y $\beta$, comprobar analíticamente las condiciones numéricas (11d)-(11f) resolviendo respectivamente:

$$
\begin{array}{llll}
\min _{F} & f(F) & \text { s.a.: } & \underline{\mathrm{F}} \leq F \leq \overline{\mathrm{F}} \\
\min _{F} & g(F) & \text { s.a.: } & \underline{\mathrm{F}} \leq F \leq \overline{\mathrm{F}} \\
\min _{F} & g \circ f(F) & \text { s.a.: } & \underline{\mathrm{F}} \leq F \leq \overline{\mathrm{F}}
\end{array}
$$

Denótese por $F^{*}$ a la solución de cualquiera de los problemas anteriores. Si $F^{*}=\overline{\mathrm{F}}$ para todos, entonces el modelo obtenido es monótono decreciente (coherente con la física del proceso) y el algoritmo termina. En otro caso, añadir cada valor $F^{*} \neq \overline{\mathrm{F}}$ donde corresponda en el conjunto ordenado $\mathcal{N}$ y volver al paso 1 .

\section{IMPLEMENTACIÓN RTO Y RESULTADOS}

El problema (8) se ha codificado en CasADi (entorno con interfaz MATLAB) y resuelto utilizando el algoritmo IPOPT [4]. Sin embargo, para la implementación se han de tener en cuenta las siguientes consideraciones.

En primer lugar, puesto que los modelos experimentales se han ajustado con datos en un determinado rango de operación, si los caudales que haya en los evaporadores en tiempo real, $\hat{\mathrm{F}}_{e}$, están fuera de dicho rango, la optimización no se debe realizar. Esto se debe a que para calcular el coeficiente de ensuciamiento $K_{\mathrm{f}}$ mediante (4) se necesita haber calculado previamente la temperatura de salida que predice el modelo (con el caudal actual medido $\hat{F}$ ). Por tanto, un caudal fuera de rango puede corresponder a un valor erróneo de temperatura si el modelo falla al extrapolar, error que se transmite directamente a la estimación de $\mathrm{K}_{\mathrm{f}}$.

En el caso de que algún evaporador esté en mantenimiento en el momento de ejecutar la optimización, es decir, no este procesando ningún producto, el caudal de agua que recibe ese condensador (mantenimiento) queda fijo en la optimización. Para ello, se impone que si la carga de un evaporador (EC) es menor a 1, no se tengan en cuenta los limites de caudal en ese condensador y que el caudal óptimo sea igual al medido en ese momento, es decir, la restricción (8e) se vería reemplazada por las siguientes expresiones:

$$
\begin{aligned}
\underline{\mathrm{F}_{e} \leq F_{e}} \leq \overline{\mathrm{F}_{e}} \quad \forall e \in\left\{e \mid \mathrm{EC}_{e}>1\right\} \\
F_{e}=\hat{\mathrm{F}}_{e} \quad \forall e \notin\left\{e \mid \mathrm{EC}_{e}>1\right\}
\end{aligned}
$$

Por último, puede darse el caso que, debido al estado de ensuciamiento y/o a la temperatura de entrada de agua a los condensadores, alguna potencia de refrigeración calculada por la optimización se encuentre fuera del rango en el que se han obtenido los modelos experimentales (7), por lo que la optimalidad no está garantizada en la práctica.

Puesto que el objetivo de esta herramienta es ayudar en la toma de decisión, se ha decidido mostrar una advertencia que informa al usuario en el caso de darse estas situaciones.

\section{RESULTADOS}

La herramienta para optimizar la distribución de agua ya está implementada y actualmente se encuentra en periodo de pruebas en Lenzing AG. En las figuras 8 - 10 se muestra un ejemplo de los resultados obtenidos a partir de unos datos reales de muestra. Nótese que dichos datos corresponden al estado particular de la red en un istante de tiempo 
cualquiera, por lo que los beneficios obtenidos por la optimización no tienen por qué ser representativos de la operación media a largo plazo.

En primer lugar, comparando los caudales optimizados con los reales medidos (Figura 8), se observa que la mayoría de plantas deben aumentar el consumo de agua. Puesto que el agua de refrigeración tiene un coste, esto puede resultar incoherente a priori. Sin embargo, como se observa en la Figura 9 , el consumo de vapor ha disminuido sensiblemente, y su coste es 10 veces mayor que el del agua ${ }^{3}$, por lo que la suma de ambos costes con la nueva distribución reporta un ahorro en coste total respecto a la situación actual.

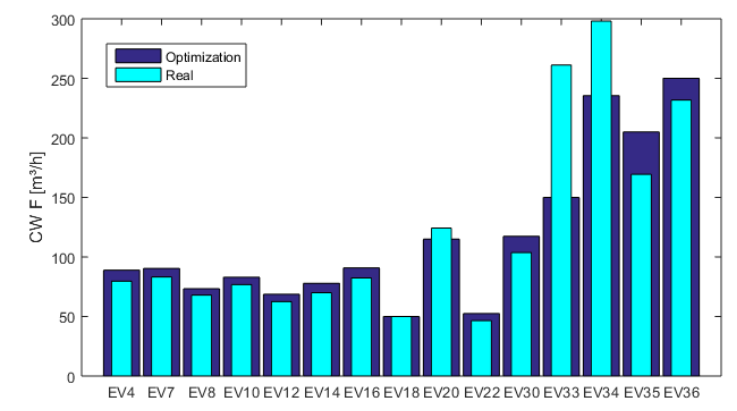

Figura 8: Resultados de caudales de refrigeración

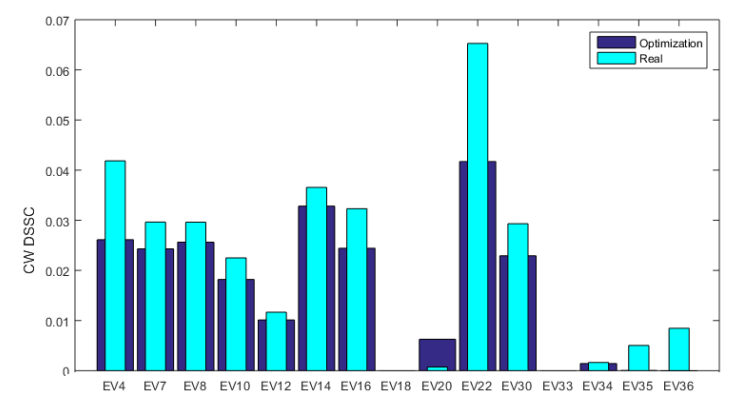

Figura 9: Resultados de consumo de vapor

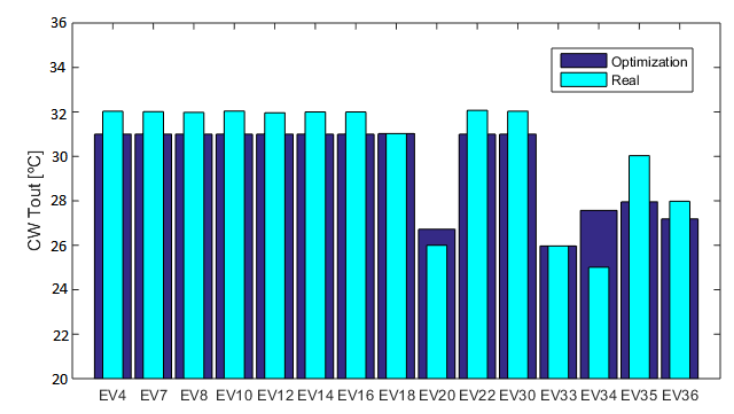

Figura 10: Resultados de temperatura

Nótese que el incremento en el consumo de vapor de las plantas 18 y 33 es nulo (Figura 9). Esto se

\footnotetext{
${ }^{3}$ Los valores reales se omiten por razones de confidencialidad con la empresa.
}

debe a que los datos experimentales obtenidos para ajustar el modelo (7) no son consistentes, por lo que se ha asumido que el consumo de vapor de esos evaporadores es un valor fijo. Así pues, los caudales para estos evaporadores se eligen simplemente con un criterio de factibilidad: cumplir la restricción de temperatura de salida máxima.

Por otra parte, analizando los resultados de temperatura de la Figura 10, se observa como en los datos medidos la temperatura de salida del refrigerante sobrepasa la máxima permitida en la mayoría de las plantas. Sin embargo, en la solución obtenida tras la optimización, los caudales se han ajustado para que dicha temperatura esté siempre por debajo del límite impuesto, cumpliendo así las restricciones medioambientales.

Por último, en la Tabla 1 se muestran los costes del sistema de refrigeración antes y después de la optimización, así como el ahorro en costes que se obtendría.

Tabla 1: Costes y ahorro

\begin{tabular}{|c|c|c|}
\hline Coste sin optimizar & $71,39 € / \mathrm{h}$ & $625,38 \mathrm{k} € /$ year \\
\hline Coste optimizado & $19,88 € / \mathrm{h}$ & $174,15 \mathrm{k} € /$ year \\
\hline Ahorro & $51,51 € / \mathrm{h}$ & $451,23 \mathrm{k} € /$ year \\
\hline
\end{tabular}

Extrapolando estos resultados se puede predecir un considerable ahorro anual que se obtendría aplicando las recomendaciones de la herramienta RTO desarrollada. No obstante, téngase en cuenta que estos valores solo representan un instante de tiempo particular y no la operación media a lo largo de un año, por lo que únicamente indican que existe un potencial márgen de mejora.

\section{CONCLUSIONES Y TRABAJO FUTURO}

En este trabajo se ha abordado un problema de modelado y optimización en un sistema de refrigeración industrial. Los conceptos de presentados en este documento han servido para desarrollar una herramienta que ayude a la toma de decisiones en tiempo real.

En primer lugar, a partir de datos experimentales se han desarrollado modelos de caja negra que relacionan las variables más importantes del sistema. Una metodología de dos pasos basada en programación mixta-entera no lineal permite ajustar tanto los parámetros como la complejidad de dichos modelos de forma simultánea, haciendo cumplir la fisica del proceso. Además, el estado de ensuciamiento de los equipos se puede estimar a partir de medidas en tiempo real, adaptando así los 
modelos a la operación actual. Con los modelos, la herramienta RTO resuelve un problema NLP de acuerdo a las limitaciones de producción actuales y al estado de los equipos. Sin embargo, no se incluye predicción alguna de la degradación por ensuciamiento, por lo que las decisiones propuestas podrían ser subóptimas a largo plazo.

Cabe destacar que la herramienta RTO desarrollada ya se encuentra en periodo de evaluación en Lenzing AG: la implementación en el sistema se está llevando paso a paso para comprobar la eficacia de la herramienta con más tests en línea y para asegurar la aceptabilidad del personal de la planta. Se requiere alrededor de un año de operación normal para evaluar el impacto, pero las pruebas preliminares realizadas con los datos mostrados anteriormente revelan ahorros de alrededor de $400 \mathrm{k} € /$ año.

\section{Agradecimientos}

Este trabajo ha sido financiado por el programa H2020 de la Unión Europea (contrato no. 723575) y el Gobierno de España (MINECO/FEDER DPI2015-70975-P).

\section{English summary}

\section{MODELING FOR OPTIMAL OPE- RATION OF AN INDUSTRIAL COOLING SYSTEM}

\begin{abstract}
This work deals with the problem of optimal distribution of cooling water through a network of evaporation plants. The end goal is developing an RTO tool to balance the water usage with the energy consumption in the plants (live steam). The optimization problem uses experimental models to assess the impact of the cooling system operation into the global plants efficiency. An effective modeling methodology for optimization purposes is proposed such that models physical coherence is ensured. Moreover, the tool provides a periodic estimation of the equipment performance degradation due to continuous operation (fouling in tube heat exchangers).
\end{abstract}

Keywords: evaporation plants, water distribution, black-box models, RTO.

\section{Referencias}

[1] S. Krämer and S. Engell, Resource Efficiency of Processing Plants: Monitoring and Improvement. John Wiley \& Sons, 2017.

[2] C. de Prada, D. Sarabia, G. Gutierrez, E. Gomez, S. Marmol, M. Sola, C. Pascual, and
R. Gonzalez, "Integration of RTO and MPC in the hydrogen network of a petrol refinery," Processes, vol. 5, no. 1, 2017.

[3] C. de Prada and J. L. Pitarch, "Real-time optimization (RTO) systems," in Resource Efficiency of Processing Plants: Monitoring and Improvement, S. Krämer and S. Engell, Eds. John Wiley \& Sons, 2017, ch. 11, pp. 265-292.

[4] J. Andersson, J. Åkesson, and M. Diehl, "Casadi: A symbolic package for automatic differentiation andoptimal control," in Recent Advances in Algorithmic Differentiation, S. Forth, P. Hovland, E. Phipps, J. Utke, and A. Walther, Eds. Berlin, Heidelberg: Springer Berlin Heidelberg, 2012, pp. 297-307.

[5] "OSIsoft PI system," https://www.osisoft. com/corporate/connected-services/pisystem. html [Online; accessed 15-June-2018].

[6] C. de Prada, D. Hose, G. Gutierrez, and J. L. Pitarch, "Developing grey-box dynamic process models," IFAC-PapersOnLine, vol. 51, no. 2, pp. 523 - 528, 2018, 9th Vienna Int. Conference on Mathematical Modelling.

[7] J. L. Pitarch, C. G. Palacín, A. Merino, and C. de Prada, "Optimal operation of an evaporation process," in Modeling, Simulation and Optimization of Complex Processes HPSC 2015, H. G. Bock, H. X. Phu, R. Rannacher, and J. P. Schlöder, Eds. Cham: Springer International Publishing, 2017, pp. 189-203.

[8] C. G. Palacín, J. L. Pitarch, C. Jasch, C. A. Méndez, and C. de Prada, "Robust integrated production-maintenance scheduling for an evaporation network," Computers $\&$ Chemical Eng., vol. 110, pp. 140 - 151, 2018.

[9] A. Cozad, N. V. Sahinidis, and D. C. Miller, "Learning surrogate models for simulationbased optimization," AIChE Journal, vol. 60, no. 6, pp. 2211-2227, 2014.

[10] C. M. Hurvich and C. L. Tsai, "A corrected Akaike information criterion for vector autoregressive model selection," J. of Time Series Analysis, vol. 14, no. 3, pp. 271-279, 1993. 